\title{
Linear seesaw model with hidden gauge symmetry
}

\author{
Takaaki Nomura, ${ }^{1, *}$ and Hiroshi Okada ${ }^{2, \dagger}$ \\ ${ }^{1}$ School of Physics, KIAS, Seoul 02455, Republic of Korea \\ ${ }^{2}$ Asia Pacific Center for Theoretical Physics, Pohang, Geyoengbuk 790-784, Republic of Korea
}

(Received 4 July 2018; published 25 March 2019)

\begin{abstract}
We propose a natural realization of a linear seesaw model with hidden gauge symmetry in which $S U(2)_{L}$ triplet fermions and one extra Higgs singlet, doublet, and quartet scalar are introduced. Small neutrino mass can be realized by two suppression factors that are the small vacuum expectation value of a quartet scalar and the inverse of a Dirac mass for the triplet. After formulating the neutrino mass matrix, we discuss the collider phenomenology of the model, focusing on signals from exotic charged particles production at the LHC.
\end{abstract}

DOI: $10.1103 /$ PhysRevD.99.055033

\section{INTRODUCTION}

One of the big mysteries in the standard model (SM) of particle physics is the mass spectrum and flavor structure of fermions. In particular, existence of physics beyond the SM is required from at least two nonzero neutrino masses for its generating mechanism. Moreover, the neutrino mass indicates a hint of structure of new physics as it should explain the smallness of the mass. Actually, many mechanisms to generate neutrino mass are proposed, such as a canonical seesaw model [1-4], inverse seesaw model [5,6], linear seesaw model [6-8], etc. Note here that mass hierarchies in the neutral mass matrix are always assumed in order to get sizable neutrino mass. Thus, appropriate explanations about these hierarchies are also one of the important tasks in our models and there exist several explanations $[9,10]$. In light of the motivation, one interesting scenario is to generate neutrino mass using the exotic fields, which are large $S U(2)_{L}$ multiplets like quartet, quintet, or septet [11-17], since we can suppress neutrino mass by the small vacuum expectation value (VEV) of a large multiplet scalar and/or restricted structure of interactions, including large multiplet fields. Furthermore, this kind of scenario would induce interesting phenomenology at collider experiments, since a large multiplet field contains multicharged particles, such as doubly charged scalars and fermions.

In this paper, we propose a natural realization of the linear seesaw model with hidden gauge symmetry in which

\footnotetext{
*nomura@kias.re.kr

† macokada.hiroshi@apctp.org
}

Published by the American Physical Society under the terms of the Creative Commons Attribution 4.0 International license. Further distribution of this work must maintain attribution to the author(s) and the published article's title, journal citation, and DOI. Funded by SCOAP ${ }^{3}$.
$S U(2)_{L}$ triplet fermions and one extra Higgs singlet, doublet, and quartet scalar are introduced. Interestingly, tiny neutrino mass is realized by two suppression effects: the inverse of Dirac mass for the triplet fermion and small VEV of the quartet scalar, which is required by the constraint from the $\rho$ parameter, where the quartet VEV is induced in a similar way to the Higgs triplet model [18]. We formulate the neutrino mass matrix and estimate the typical size of Yukawa coupling constants associated with triplet fermion and SM leptons. Then we discuss collider phenomenology of our scenario, focusing on production of exotic charged particles at the large hadron collider (LHC). Particularly interesting signals come from Yukawa interaction associated with triplet fermion, quartet scalar, and SM lepton, which represent a specific signature of our model.

This paper is organized as follows. In Sec. II, we introduce our model and formulate the Higgs sector, neutral gauge sector, neutrino sector, and relevant interactions. In Sec. III, we discuss collider phenomenologies of exotic charged particles considering the specific signature in our model. Finally, we summarize our results and give the conclusion in Sec. IV.

\section{MODEL SETUP AND CONSTRAINTS}

In this section, we formulate our model, introducing hidden gauge symmetry $U(1)_{H}$. At first, we add three families of $S U(2)_{L}$ triplet right(left)-handed fermions $\Sigma_{R}\left(\Sigma_{L}\right)$ with $U(1)_{H}$ charge 1 ; the triplet fermions can satisfy anomaly cancellation conditions since they are vectorlike. In the scalar sector, we introduce three new scalar fields in addition to the SM Higgs field, which are $S U(2)_{L}$ doublet $H_{1}, S U(2)_{L}$ quartet $H_{4}$, and SM singlet $\varphi$ with $U(1)_{H}$ charge 1 . Here we denote each VEV of the scalar fields to be $\langle H\rangle \equiv v_{H} / \sqrt{2},\left\langle H_{1}\right\rangle \equiv v_{1} / \sqrt{2},\left\langle H_{4}\right\rangle \equiv$ $v_{4} / \sqrt{2}$, and $\langle\Phi\rangle \equiv v_{\Phi} / \sqrt{2}$, where $H$ is expected to be the 
TABLE I. Charge assignments of our fields under $S U(3)_{C} \times$ $S U(2)_{L} \times U(1)_{Y} \times U(1)_{H}$, where the lower index $a$ is the number of family that runs over $1-3$.

\begin{tabular}{lcccrrrrrrrr}
\hline \hline & $Q_{L_{a}}$ & $u_{R_{a}}$ & $d_{R_{a}}$ & $L_{L_{a}}$ & $e_{R_{a}}$ & $\Sigma_{R_{a}}$ & $\Sigma_{L_{a}}$ & $H$ & $H_{1}$ & $H_{4}$ & $\Phi$ \\
\hline$S U(3)_{C}$ & $\mathbf{3}$ & $\mathbf{3}$ & $\mathbf{3}$ & $\mathbf{1}$ & $\mathbf{1}$ & $\mathbf{1}$ & $\mathbf{1}$ & $\mathbf{1}$ & $\mathbf{1}$ & $\mathbf{1}$ & $\mathbf{1}$ \\
$S U(2)_{L}$ & $\mathbf{2}$ & $\mathbf{1}$ & $\mathbf{1}$ & $\mathbf{2}$ & $\mathbf{1}$ & $\mathbf{3}$ & $\mathbf{3}$ & $\mathbf{2}$ & $\mathbf{2}$ & $\mathbf{4}$ & $\mathbf{1}$ \\
$U(1)_{Y}$ & $\frac{1}{6}$ & $\frac{2}{3}$ & $-\frac{1}{3}$ & $-\frac{1}{2}$ & -1 & 0 & 0 & $\frac{1}{2}$ & $\frac{1}{2}$ & $-\frac{1}{2}$ & 0 \\
$U(1)_{H}$ & 0 & 0 & 0 & 0 & 0 & 1 & 1 & 0 & 1 & 1 & 1 \\
\hline \hline
\end{tabular}

SM-like Higgs. All the field contents and their charge assignments are summarized in Table I.

We write the singlet and doublet scalar fields by

$$
\begin{aligned}
\Phi & =\frac{1}{\sqrt{2}}\left(v_{\Phi}+\phi+i \eta_{\Phi}\right), \quad H=\left(\begin{array}{c}
h^{+} \\
\frac{v_{H}+\tilde{h}+i \eta}{\sqrt{2}}
\end{array}\right), \\
H_{1} & =\left(\begin{array}{c}
h_{1}^{+} \\
\frac{v_{1}+h_{1}+i \eta_{1}}{\sqrt{2}}
\end{array}\right) .
\end{aligned}
$$

The quartet scalar $H_{4}$ with hypercharge $Y=-1 / 2$ is represented as

$$
H_{4}=\left(\varphi_{1}^{+}, \varphi^{0}, \varphi_{2}^{-}, \varphi^{--}\right)^{T} \quad \text { or } \quad\left(H_{4}\right)_{i j k}
$$

where subscripts for a singly charged component distinguish two independent fields, and $\left(H_{4}\right)_{i j k}$ is the symmetric tensor notation, with $S U(2)_{L}$ index $\{i, j, k\}$ taking 1 or 2 , defined by $\left(H_{4}\right)_{[111]}=\varphi_{1}^{+},\left(H_{4}\right)_{[112]}=\varphi^{0} / \sqrt{3},\left(H_{4}\right)_{[122]}=$ $\varphi_{2}^{-} / \sqrt{3}$, and $\left(H_{4}\right)_{[222]}=\varphi^{--} ;[i j k]$ indicates symmetric indices under exchange among them. Note also that the neutral component is written by $\varphi^{0}=\left(v_{4}+\varphi_{R}^{0}+i \varphi_{I}^{0}\right) /$ $\sqrt{2}$. The triplet fermion with hypercharge $Y=0$ is given by

$$
\Sigma_{L_{a}\left(R_{a}\right)}=\left(\begin{array}{cc}
\frac{\Sigma^{0}}{\sqrt{2}} & \Sigma^{+} \\
\Sigma^{\prime-} & -\frac{\Sigma^{0}}{\sqrt{2}},
\end{array}\right)_{L_{a}\left(R_{a}\right)},
$$

where two charged components are distinguished as independent fermions. ${ }^{1}$ The mass of $\Sigma$ is given by Dirac type

$$
M_{\Sigma} \operatorname{Tr}[\bar{\Sigma} \Sigma]=M_{\Sigma}\left(\bar{\Sigma}^{+} \Sigma^{+}+\bar{\Sigma}^{0} \Sigma^{0}+\bar{\Sigma}^{\prime-} \Sigma^{\prime-}\right)
$$

where we have omitted the flavor index. Note that the Majorana mass term of the triplet fermions is forbidden by $U(1)_{H}$ symmetry and the type-III seesaw mechanism is absent in our setup.

\footnotetext{
${ }^{1}$ We can also write $\Sigma$ as symmetry tensor forms $\Sigma_{11}=\Sigma^{+}$, $\Sigma_{12}=\Sigma_{21}=\Sigma^{0} / \sqrt{2}$, and $\Sigma^{\prime-}=\Sigma_{22}$.
}

The relevant Yukawa Lagrangian under these symmetries is given by ${ }^{2}$

$$
\begin{aligned}
-\mathcal{L}_{\ell}= & y_{\ell_{a}} \bar{L}_{L_{a}} H e_{R_{a}}+y_{R_{a b}}\left[\bar{L}_{L_{a}} \tilde{H}_{1} \Sigma_{R_{b}}\right]+y_{L_{a b}}\left[\bar{L}_{L_{a}} H_{4} \Sigma_{L_{b}}^{c}\right] \\
& + \text { H.c., }
\end{aligned}
$$

where $\tilde{H} \equiv i \sigma_{2} H$, upper indices $(a, b)=1-3$ are the number of families, and $y_{\ell}$ and $M_{\Sigma}$ can be the diagonal matrix without loss of generality due to the redefinitions of the fermions. Here, we explicitly write our Lagrangian in terms of each of the components

$$
\begin{gathered}
y_{R_{a b}}\left[\bar{L}_{L_{a}} \tilde{H}_{1} \Sigma_{R_{b}}\right]=\frac{y_{R_{a b}}}{\sqrt{2}}\left[\bar{e}_{L_{a}}\left(\sqrt{2} \Sigma_{R_{b}}^{\prime} h_{1}^{*}+\Sigma_{R_{b}}^{0} h_{1}^{-}\right)\right. \\
\left.+\bar{\nu}_{L_{a}}\left(\Sigma_{R_{b}}^{0} h_{1}^{*}+\sqrt{2} \Sigma_{R_{b}}^{+} h_{1}^{-}\right)\right], \\
y_{L_{a b}}\left[\bar{L}_{L_{a}} H_{4} \Sigma_{L_{b}}^{c}\right] \\
=\frac{y_{L_{a b}}\left[\bar{e}_{L_{a}}\left(\sqrt{3} \Sigma_{L_{b}}^{-c} \varphi^{--}+\sqrt{2} \Sigma_{L_{b}}^{0 c} \varphi_{2}^{-}+\Sigma_{L_{b}}^{+c} \varphi^{0}\right)\right.}{\sqrt{3}} \\
\left.+\bar{\nu}_{L_{a}}\left(\sqrt{3} \Sigma_{L_{b}}^{+c} \varphi_{1}^{+}+\sqrt{2} \Sigma_{L_{b}}^{0 c} \varphi^{0}+\Sigma_{L_{b}}^{-c} \varphi_{2}^{-}\right)\right] .
\end{gathered}
$$

From these Yukawa couplings, we obtain mass matrices defined by $m_{\ell}=y_{\ell} v_{H} / \sqrt{2}, m_{D}=y_{R} v_{1} / \sqrt{2}, \delta_{D}=y_{L} v_{4} /$ $\sqrt{3}$, where $m_{D}$ and $\delta_{D}$ contribute to the neutrino mass matrix as we discuss below. In our model, we assign lepton number 1 to $\Sigma_{L, R}$ and the term with $y_{L}$ breaks lepton number conservation.

\section{A. Scalar sector}

The scalar potential of our model is

$$
\begin{aligned}
\mathcal{V}= & -\mu_{H}^{2} H^{\dagger} H+\mu_{H_{1}}^{2} H_{1}^{\dagger} H_{1}+M_{4}^{2} H_{4}^{\dagger} H_{4}-\mu_{\Phi}^{2} \Phi^{\dagger} \Phi \\
& +\mathcal{V}_{4}+\mathcal{V}_{\text {nontrivial }}, \\
\mathcal{V}_{4}= & \lambda_{H}\left(H^{\dagger} H\right)^{2}+\lambda_{H_{1}}\left(H_{1}^{\dagger} H_{1}\right)^{2}+\lambda_{H_{4}}\left(H_{4}^{\dagger} H_{4}\right)^{2} \\
& +\lambda_{H H_{1}}\left(H^{\dagger} H\right)\left(H_{1}^{\dagger} H_{1}\right)+\lambda_{H H_{4}}\left(H^{\dagger} H\right)\left(H_{4}^{\dagger} H_{4}\right) \\
& +\lambda_{H_{1} H_{4}}\left(H_{1}^{\dagger} H_{1}\right)\left(H_{4}^{\dagger} H_{4}\right),
\end{aligned}
$$

where $\mathcal{V}_{4}$ indicates trivial four point interaction terms. The parameters in $\mathcal{V}_{4}$ are assumed to satisfy constraints from unitarity and perturbativity, and we do not discuss them in our analysis since it is not closely related to neutrino mass generation and collider physics. The nontrivial scalar potential terms are given by

$$
\mathcal{V}_{\text {nontrivial }}=\lambda_{0}\left[\left(H_{4} H \tilde{H}_{1} H\right)+\text { H.c. }\right]+\mu_{0}\left[\left(H_{1}^{\dagger} H\right) \Phi+\text { H.c. }\right] \text {, }
$$

\footnotetext{
${ }^{2}$ Since the structure of the quark sector is exactly the same as the one in the SM, we neglect it hereafter.
} 
where $S U(2)_{L}$ indices are implicitly contracted to be gauge invariant in the first term. These nontrivial terms forbid dangerous massless Goldstone bosons that would be induced from $H_{1,4}$ after symmetry breaking. The VEVs of the scalar fields are obtained by imposing the condition $\partial \mathcal{V} / \partial v_{H, 1,4, \Phi}=0$, where we assume $M_{4}^{2}>0$ in the potential. Then $v_{4}$ is roughly given by

$$
\begin{aligned}
v_{4} \sim \frac{\lambda_{0} v_{1} v_{H}^{2}}{M_{4}^{2}}= & \lambda_{0}\left(\frac{v_{1}}{100 \mathrm{GeV}}\right)\left(\frac{v_{H}}{100 \mathrm{GeV}}\right)^{2} \\
& \times\left(\frac{1000 \mathrm{GeV}}{M_{4}}\right)^{2} \mathrm{GeV} .
\end{aligned}
$$

This VEV is restricted by the $\rho$ parameter, which is given by

$$
\rho=\frac{v_{H}^{2}+v_{1}^{2}+7 v_{4}^{2}}{v_{H}^{2}+v_{1}^{2}+v_{4}^{2}}
$$

where the experimental value is $\rho=1.0004_{-0.0004}^{+0.0003}$ at $2 \sigma$ confidence level [19]. On the other hand, we also require $v \equiv v_{H}^{2}+v_{1}^{2}+7 v_{4}^{2}=1 /\left(\sqrt{2} G_{F}\right) \approx(246 \mathrm{GeV})^{2}$. To satisfy both of conditions, one finds $v_{4} \lesssim 2.65 \mathrm{GeV}$, while $v_{1}$ can be comparable to $v_{H}$. Remarkably, we can naturally realize $v_{4} \lesssim \mathcal{O}(1) \mathrm{GeV}$ if the mass scale of the scalar quartet is $\mathcal{O}(1) \mathrm{TeV}$ or larger. With small $v_{4}$, the scalar bosons from $\mathrm{H}_{4}$ have approximately degenerate masses, which are given by $M_{4}$. In our scenario, we assume small mixing among the scalar quartet, doublets, and singlet, and we write mass eigenstates from $H_{4}$ just as $\left\{\varphi^{ \pm \pm} \varphi_{1}^{ \pm}, \varphi_{2}^{ \pm}, \varphi_{R}^{0}, \varphi_{I}^{0}\right\}$, which are approximately the same as in Eq. (2).

Assuming small mixing between two Higgs doublet sectors and the other scalar sector, interactions associated with two Higgs doublets are approximately the same as those in the type-I two Higgs doublet model (2HDM). Then we write mass eigenstates from two Higgs doublets as $\left\{h, H, A, H^{ \pm}\right\}$, where $h$ is the SM-like Higgs, $H$ is heavy neutral Higgs, $A$ is $C P$-odd Higgs, and $H^{ \pm}$is charged Higgs. In terms of the mass eigenstates, we can write the Yukawa interactions in Eq. (7) such that

$$
\begin{aligned}
& \frac{y_{R_{a b}}}{\sqrt{2}}\left[\sqrt{2} \bar{e}_{L_{a}} \Sigma_{R_{b}}^{\prime}(\cos \alpha h+\sin \alpha H-i \cos \beta A)\right. \\
& \quad+\bar{e}_{L_{a}} \Sigma_{R_{b}}^{0} \cos \beta H^{-}+\bar{\nu}_{L_{a}} \Sigma_{R_{b}}^{0}(\cos \alpha h+\sin \alpha H \\
& \left.\quad-i \cos \beta A)+\sqrt{2} \bar{\nu}_{L_{a}} \Sigma_{R_{b}}^{+} \cos \beta H^{-}\right]
\end{aligned}
$$

where $\cos \alpha(\sin \alpha)$ correspond to mixing among neutral scalars in the two Higgs doublet, and $\tan \beta=v_{1} / v_{H}$.

After $U(1)_{H}$ symmetry breaking, the $C P$-odd component of the singlet scalar $\Phi$ is absorbed by the $Z^{\prime}$ boson as the Nambu-Goldstone boson, while the $C P$-even component is a physically neutral scalar boson. Under small mixing assumption, this $C P$-even scalar boson does not provide any interesting phenomenologies and we will not discuss it hereafter.

\section{B. $Z^{\prime}$ boson from $U(1)_{H}$}

In this model, we have a massive $Z^{\prime}$ boson from the spontaneous breaking of $U(1)_{H}$ gauge symmetry. Here we assume $Z^{\prime}$ mass is mostly induced by the VEV of singlet scalar $\Phi$ such that

$$
m_{Z^{\prime}} \simeq g_{H} v_{\Phi},
$$

where $g_{H}$ is the gauge coupling constant associated with $U(1)_{H}$. Since SM particles are not charged under the $U(1)_{H}, Z^{\prime}$ is a hidden gauge boson and it is difficult to directly produce it at collider experiments. Thus we will not discuss $Z^{\prime}$ boson physics further in this paper.

\section{Neutrino sector}

After the spontaneous symmetry breaking, neutral fermion mass matrix with $9 \times 9$ is given by

$$
M_{N}=\left[\begin{array}{ccc}
0 & m_{D} & \delta_{D} \\
m_{D}^{T} & 0 & M_{\Sigma}^{T} \\
\delta_{D}^{T} & M_{\Sigma} & 0
\end{array}\right] .
$$

Then the active neutrino mass matrix can approximately be found as

$$
m_{\nu} \approx-\delta_{D}\left(M_{\Sigma}^{T}\right)^{-1} m_{D}^{T}-m_{D} M_{\Sigma}^{-1} \delta_{D}^{T},
$$

where $\delta_{D} \ll M_{\Sigma}$ is expected. Let us estimate the neutrino mass order. If $m_{D} \approx \mathcal{O}(0.01) \mathrm{GeV}, \delta_{D} / M_{\Sigma} \approx \mathcal{O}\left(10^{-8}\right)$ is expected to find the sizable neutrino masses; $m_{\nu} \sim$ $10^{-10} \mathrm{GeV}$. Moreover, in terms of the Yukawa coupling constant and VEVs, we can write

$$
m_{\nu} \sim y_{L} y_{R} \frac{v_{1} v_{4}}{M_{\Sigma}} .
$$

Taking $M_{\Sigma}=1000 \mathrm{GeV}, v_{4}=1 \mathrm{GeV}$, and $v_{1} \lesssim 100 \mathrm{GeV}$, we can realize $m_{\nu} \lesssim 10^{-10} \mathrm{GeV}$ with $y_{L} \sim y_{R} \lesssim 10^{-4}$, which is similar in magnitude to those in generating SM charged leptons.

The neutrino mass matrix is diagonalized by unitary matrix $U_{\mathrm{MNS}} ; D_{\nu}=U_{\mathrm{MNS}}^{T} m_{\nu} U_{\mathrm{MNS}}$, where $D_{\nu} \equiv \operatorname{diag}\left(m_{1}\right.$, $\left.m_{2}, m_{3}\right)$. One of the elegant ways to reproduce the current neutrino oscillation data [19] is to apply the Casas-Ibarra parametrization [20] and find the following relation:

$$
m_{D}=-\frac{1}{2} \delta_{D}\left(M_{\Sigma}^{T}\right)^{-1}\left(U_{\mathrm{MNS}}^{*} \sqrt{D_{\nu}} U_{\mathrm{MNS}}^{\dagger}+A\right) .
$$

Here $A$ is an arbitrary $3 \times 3$ antisymmetric matrix with complex value; $A+A^{T}=0$. Note here that all the 
components of $m_{D}$ should not exceed $100 \mathrm{GeV}$, once the perturbative limit of $y_{R}$ is taken to be one.

\section{Nonunitarity}

Constraint of nonunitarity should always be taken into account in case of a larger neutral mass matrix whose components are greater than $3 \times 3$, since experimental neutrino oscillation results suggest nearly unitary. In case of the linear seesaw, when nonunitarity matrix $U_{\mathrm{MNS}}^{\prime}$ is defined, one can typically parametrize it by the following form:

$$
U_{\mathrm{MNS}}^{\prime} \equiv\left(1-\frac{1}{2} F F^{\dagger}\right) U_{\mathrm{MNS}}
$$

where $F \equiv\left(M_{\Sigma}^{T}\right)^{-1} m_{D}$ is a Hermitian matrix, and $U_{\text {MNS }}^{\prime}$ represents the deviation from the unitarity. Considering several experimental bounds [21], one finds the following constraints [22]:

$$
\left|F F^{\dagger}\right| \leq\left[\begin{array}{lll}
2.5 \times 10^{-3} & 2.4 \times 10^{-5} & 2.7 \times 10^{-3} \\
2.4 \times 10^{-5} & 4.0 \times 10^{-4} & 1.2 \times 10^{-3} \\
2.7 \times 10^{-3} & 1.2 \times 10^{-3} & 5.6 \times 10^{-3}
\end{array}\right] .
$$

Here, we show a benchmark point to satisfy the neutrino oscillation data [23], nonunitarity constraints, and perturbativity $y_{R} \lesssim 1$, within our parameter choices. Fixing the following values $\left(v_{1}, v_{4}\right)=(1,100) \mathrm{GeV}$, the benchmark points are given by

$$
\begin{aligned}
\left(A_{12}, A_{13}, A_{23}\right) & \left(-0.0306,-8.34 \times 10^{-5}, 5.93 \times 10^{-4}\right) \mathrm{GeV}, \\
m_{D} \approx & {\left[\begin{array}{ccc}
-1.6+0.019 i & -478-0.007 i & -18-0.01 i \\
15.8-0.17 i & 9654+0.27 i & -30.1+0.27 i \\
-56+0.60 i & -27830-0.75 i & -4.26-0.77 i
\end{array}\right] } \\
& \times 10^{-10} \mathrm{GeV}, \\
M_{\Sigma} \approx & {\left[\begin{array}{ccc}
1.48 & 1.50 & 1.95 \\
1.13 & 1.44 & 1.65 \\
1.04 & 1.46 & 1.37
\end{array}\right] \times 10^{3} \mathrm{GeV}, } \\
\delta_{D} \approx & {\left[\begin{array}{ccc}
0.367 & 1.70 & 2.57 \\
5.51 & 0.50 & 12.5 \\
18.04 & 32.8 & 0.83
\end{array}\right] \times 10^{-3} \mathrm{GeV}, }
\end{aligned}
$$

where we assumed real elements of $A$ and the normal neutrino mass ordering with vanishing neutrino mass for the lightest neutrino, for simplicity. Even though one analyzes it with a general framework, reproducing neutrino oscillation data with these constraints can easily be achieved due to enough input parameters.

\section{Lepton flavor violations (LFVs) and charged-lepton mass matrix}

Since $y_{R}$ is expected to be small from the previous discussion, we focus on the Yukawa term $y_{L}$ that gives rise to $\mu \rightarrow e \gamma$ processes at the one-loop level, which is the most stringent constraint from the MEG experiment [24]; therefore, its branching ratio is given by $B(\mu \rightarrow e \gamma) \leq$ $4.2 \times 10^{-13}$. While our branching ratio is given by

$$
\begin{aligned}
B(\mu \rightarrow e \gamma) \approx & \frac{48 \pi^{3} \alpha_{\mathrm{em}}}{G_{\mathrm{F}}^{2}} \mid \sum_{\alpha=1-3} \frac{y_{L_{1 \alpha}} y_{L_{\alpha 2}}^{\dagger}}{(4 \pi)^{2}}\left[2 F\left(\Sigma_{\alpha}^{-}, \varphi^{--}\right)\right. \\
& \left.+F\left(\Sigma_{\alpha}^{0}, \varphi^{-}\right)\right]\left.\right|^{2}
\end{aligned}
$$

where we assume all the masses in the components of $\Sigma$ and $H_{4}$ to be degenerate, $G_{\mathrm{F}} \approx 1.166 \times 10^{-5} \mathrm{GeV}^{-2}$ is the Fermi constant, $\alpha_{\mathrm{em}}\left(m_{Z}\right) \approx 1 / 128.9$ is the fine-structure constant [19], and

$$
F\left(m_{1}, m_{2}\right) \approx \frac{m_{1}^{6}+3 m_{1}^{4} m_{2}^{2}-6 m_{1}^{2} m_{2}^{4}+m_{2}^{6}+12 m_{1}^{4} m_{2}^{2} \ln \left[\frac{m_{2}}{m_{1}}\right]}{12\left(m_{1}^{2}-m_{2}^{2}\right)^{4}} .
$$

Comparing our branching ratio with the experimental one, one finds the following bounds on Yukawa couplings:

$$
\sum_{\alpha=1-3} y_{L_{1 \alpha}} y_{L_{\alpha 2}}^{\dagger} \lesssim 2.02 \times 10^{-3}
$$

where we fix $M_{\Sigma}=600 \mathrm{GeV}$ and $m_{\varphi}=1000 \mathrm{GeV}$.

\section{Charged-lepton mass matrix}

Next, we discuss the charged-lepton mixing that is also restricted by the current experimental data. Similar to the case of LFVs, we neglect the contribution to $y_{R}$ because it is sufficiently small. Furthermore, we assume the mass matrices $m_{D}$ and $M_{\Sigma}$ to be diagonal for simplicity. Then, one finds the charged-lepton fermion mass matrix as

$$
\begin{gathered}
\left(\begin{array}{c}
\bar{e}_{L}^{a} \\
\bar{\Sigma}_{L}^{a}
\end{array}\right)^{T} M_{E}\left(\begin{array}{c}
e_{R}^{a} \\
\Sigma_{R}^{a}
\end{array}\right)=\left(\begin{array}{c}
\bar{e}_{L}^{a} \\
\bar{\Sigma}_{L}^{a}
\end{array}\right)^{T}\left[\begin{array}{cc}
m_{\ell_{a}} & \delta_{D_{a}} / \sqrt{2} \\
0 & M_{\Sigma_{a}}
\end{array}\right]\left(\begin{array}{c}
e_{R}^{a} \\
\Sigma_{R}^{a}
\end{array}\right), \\
M_{E} M_{E}^{\dagger}=\left[\begin{array}{cc}
m_{\ell_{a}}^{2} & \delta_{D_{a}} M_{\Sigma_{a}} / \sqrt{2} \\
\delta_{D_{a}} M_{\Sigma_{a}} / \sqrt{2} & M_{\Sigma_{a}}^{2}
\end{array}\right]
\end{gathered}
$$

The mass matrix is diagonalized by transformation $\left(e_{L(R)}, E_{L(R)}\right) \rightarrow V_{L(R)}^{\dagger}\left(e_{L(R)}, E_{L(R)}\right)$. Thus one obtains diagonalization matrices $V_{L}$, which diagonalizes $M_{E} M_{E}^{\dagger}$ as $V_{L} M_{E} M_{E}^{\dagger} V_{L}^{\dagger} \sim \operatorname{diag}\left(m_{\ell}^{2}, M_{\Sigma}^{2}\right)$ such that 


$$
V_{L}=\left(\begin{array}{cc}
\cos \theta & -\sin \theta \\
\sin \theta & \cos \theta
\end{array}\right), \quad \tan 2 \theta \simeq \frac{\sqrt{2} \delta_{D}}{M_{\Sigma}}
$$

where the current experimental data at LHC and large electron positron collider suggest that the lower bound on the heavier is about $M_{\Sigma} \sim 100 \mathrm{GeV}$ [19], while $\delta_{D} \sim$ $\mathcal{O}\left(10^{-10}\right) \sim \mathcal{O}\left(10^{-6}\right)$. It implies that $\theta \approx \mathcal{O}\left(10^{-12}\right) \sim$ $\mathcal{O}\left(10^{-8}\right)$, which is negligibly small.

\section{COLLIDER PHENOMENOLOGY OF THE MODEL}

In this section, we discuss production of exotic particles in the model at the LHC. Signals of our exotic particles are explored by estimating production cross section and formulating branching ratios. In particular, we focus on charged particles in quartet scalar and triplet fermions since they induce the specific signature of the model.

\section{A. Production cross sections}

The components of quartet scalar and triplet fermions can be produced by electroweak interaction at the LHC. For quartet scalar $H_{4}$, gauge interactions are derived from the kinetic term

$$
\begin{aligned}
\left|D_{\mu} H_{4}\right|^{2} \supset & \sum_{m=-\frac{3}{2},-\frac{1}{2}, \frac{1}{2}, \frac{3}{2}} \mid\left[\partial_{\mu}-i\left(-\frac{1}{2}+m\right) e A_{\mu}\right. \\
& \left.-i \frac{g}{c_{W}}\left(m-\left(-\frac{1}{2}+m\right) s_{W}^{2}\right) Z_{\mu}\right]\left(H_{4}\right)_{m} \\
& +\frac{i g}{\sqrt{2}} \sqrt{\left(\frac{3}{2}+m\right)\left(\frac{5}{2}-m\right)} W_{\mu}^{+}\left(H_{4}\right)_{m-1} \\
& +\left.\frac{i g}{\sqrt{2}} \sqrt{\left(\frac{3}{2}-m\right)\left(\frac{5}{2}+m\right)} W_{\mu}^{-}\left(H_{4}\right)_{m+1}\right|^{2},
\end{aligned}
$$

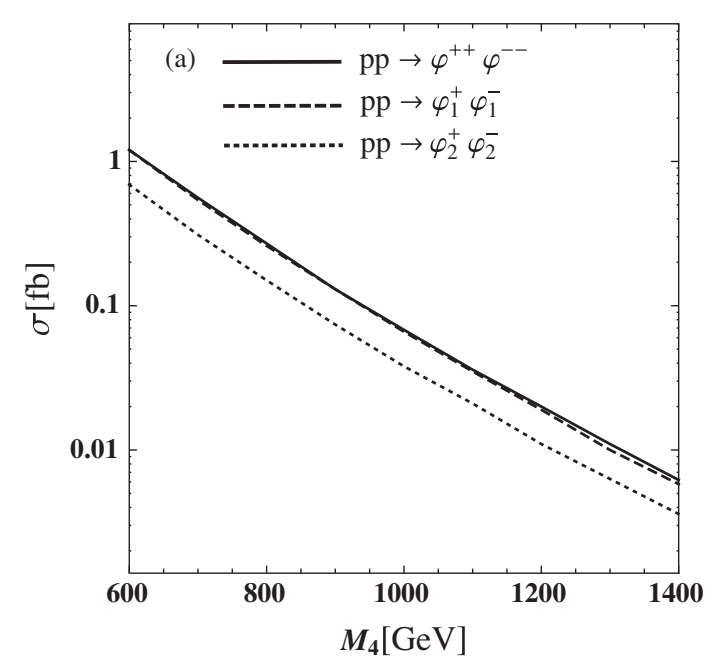

where $g$ is the gauge coupling for $S U(2)_{L}, e$ is the electromagnetic coupling constant, $s_{W}\left(c_{W}\right)=$ $\sin \theta_{W}\left(\cos \theta_{W}\right)$ with the Weinberg angle $\theta_{W}$, and $\left(H_{4}\right)_{m}$ indicates the component of $\mathrm{H}_{4}$ that has the eigenvalue of diagonal $S U(2)_{L}$ generator $T_{3}$ given by $m ;\left\{\left(H_{4}\right)_{3 / 2}\right.$, $\left.\left(H_{4}\right)_{1 / 2},\left(H_{4}\right)_{-1 / 2},\left(H_{4}\right)_{-3 / 2}\right\}=\left\{\varphi_{1}^{+}, \varphi^{0}, \varphi_{2}^{-}, \varphi^{--}\right\}$. For the triplet fermion, we explicitly write gauge interactions such that

$$
\begin{aligned}
\mathcal{L}= & g W_{\mu}^{+}\left(\bar{\Sigma}^{+} \gamma^{\mu} \Sigma^{0}+\bar{\Sigma}^{0} \gamma^{\mu} \Sigma^{\prime-}\right) \\
& +g W_{\mu}^{-}\left(\bar{\Sigma}^{0} \gamma^{\mu} \Sigma^{+}+\bar{\Sigma}^{\prime} \gamma^{\mu} \Sigma^{0}\right) \\
& +g c_{W} Z_{\mu}\left(\bar{\Sigma}^{+} \gamma^{\mu} \Sigma^{+}-\bar{\Sigma}^{\prime-} \gamma^{\mu} \Sigma^{\prime-}\right) \\
& +e A_{\mu}\left(\bar{\Sigma}^{+} \gamma^{\mu} \Sigma^{+}-\bar{\Sigma}^{\prime-} \gamma^{\mu} \Sigma^{\prime-}\right) .
\end{aligned}
$$

The exotic charged particles can be produced via interactions with the SM gauge bosons. The production cross sections are estimated using CALCHEP [25] with the CTEQ6 parton distribution functions [26]. In Fig. 1 we show cross sections for the pair production of exotic charged particles at the LHC $13 \mathrm{TeV}$. We find that production cross section for $\Sigma^{ \pm}\left(\Sigma^{\prime \pm}\right)$ pair production is larger than those for charged scalars in quartet when the mass scale is same. For $\mathcal{O}(1) \mathrm{TeV}$ mass of exotic fermions, we can obtain production cross section $\sim 1 \mathrm{fb}$, which can give a sizable number of events at the LHC.

\section{B. Decay branching ratios of exotic particles}

Here, we consider decay processes of exotic charged particles and estimate their branching ratios (BRs).

First, we consider the decay of charged scalar bosons from quartet. Partial decay width for the processes including $\Sigma$ 's in final state are given by

$$
\Gamma_{\varphi^{++} \rightarrow \ell^{+} \Sigma^{++}} \simeq \Gamma_{\varphi_{1}^{+} \rightarrow L \Sigma^{+}} \simeq \frac{y_{L}^{2}}{16 \pi} M_{4}\left(1-\frac{m_{\Sigma}^{2}}{M_{4}^{2}}\right)^{2}
$$

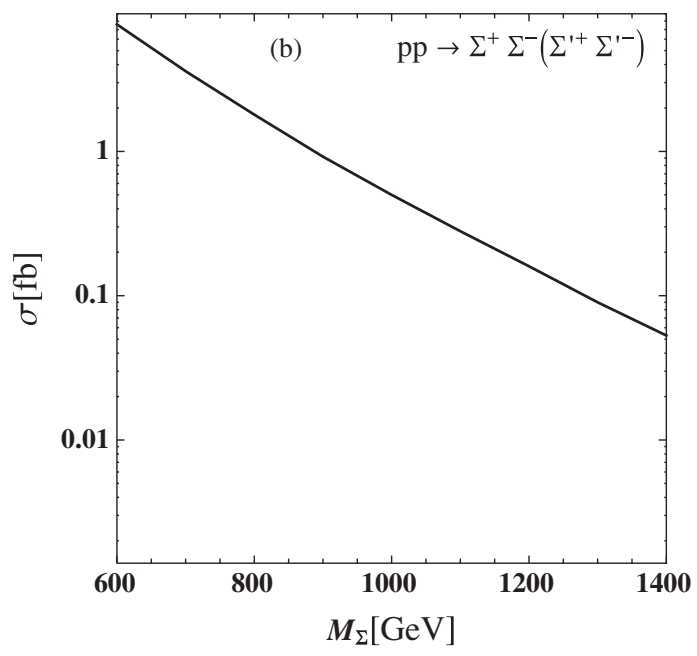

FIG. 1. (a) The cross section for pair production process $p p \rightarrow Z / \gamma \rightarrow \varphi^{++} \varphi^{--}$and $p p \rightarrow Z / \gamma \rightarrow \varphi_{1,2}^{+} \varphi_{1,2}^{-}$as a function of quartet mass $M_{4}$. (b) The cross section for pair production process $p p \rightarrow Z / \gamma \rightarrow \Sigma^{+} \Sigma^{-}\left(\Sigma^{\prime+} \Sigma^{\prime-}\right)$ as a function of triplet fermion mass $M_{\Sigma}$. 

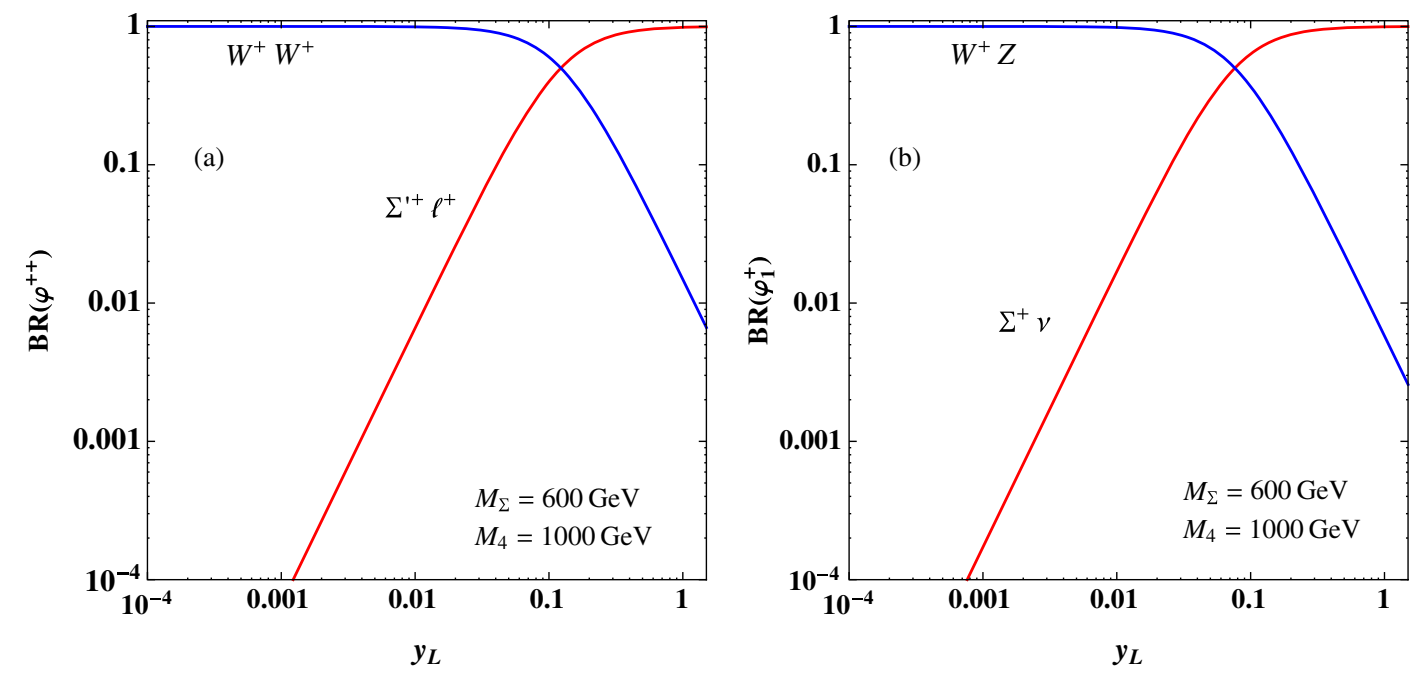

FIG. 2. (a) Branching ratio for decay of $\varphi^{++}$in quartet scalar. (b) Branching ratio for decay of $\varphi_{1}^{+}$in quartet scalar. They are given as a function of Yukawa coupling $y_{L}$, assuming one element is dominant and some parameters are fixed as indicated in the plots.

$$
2 \Gamma_{\varphi_{2}^{+} \rightarrow \ell^{+} \Sigma^{0}} \simeq \Gamma_{\varphi_{2}^{+} \rightarrow \nu \Sigma^{\prime+}}=\frac{y_{L}^{2}}{24 \pi} M_{4}\left(1-\frac{m_{\Sigma}^{2}}{M_{4}^{2}}\right)^{2}
$$

where the masses of SM leptons are ignored and we have omitted flavor index for Yukawa coupling constant $y_{L}$. Scalar bosons in quartet can also decay into two SM gauge bosons through gauge interactions

$$
\begin{aligned}
\left|D_{\mu} H_{4}\right|^{2} \supset & \sqrt{\frac{3}{2}} v_{4} W^{+} W^{+} \varphi^{--}+\frac{g^{2} v_{4}}{c_{W}}\left[s_{W}^{2} Z_{\mu} W^{+\mu} \varphi_{2}^{-}\right. \\
& \left.+\frac{\sqrt{3}}{2} c_{W}^{2} Z_{\mu} W^{+\mu} \varphi_{1}^{-}\right]+\operatorname{egv}_{4}\left[A_{\mu} W^{+\mu} \varphi_{2}^{-}\right. \\
& \left.-\frac{\sqrt{3}}{2} A_{\mu} W^{+\mu} \varphi_{1}^{-}\right]+ \text {c.c. }
\end{aligned}
$$

Then we derive partial decay widths for two gauge boson final states such that

$$
\begin{aligned}
& \Gamma_{\varphi^{++} \rightarrow W^{+} W^{+}}= \frac{3 g^{4}}{32 \pi} \frac{v_{4}^{2}}{M_{4}} \lambda^{\frac{1}{2}}\left(M_{4} ; m_{W}, m_{W}\right) \\
& \times\left[2+\frac{M_{4}^{4}}{m_{W}^{4}}\left(1-\frac{2 m_{W}^{2}}{M_{4}^{2}}\right)^{2}\right], \\
& \Gamma_{\varphi_{1}^{+} \rightarrow W^{+} Z}=\frac{3 c_{W}^{2} g^{4}}{64 \pi} \frac{v_{4}^{2}}{M_{4}} \lambda^{\frac{1}{2}}\left(M_{4} ; m_{W}, m_{Z}\right) \\
& \times\left[2+\frac{M_{4}^{4}}{m_{W}^{2} m_{Z}^{2}}\left(1-\frac{m_{W}^{2}}{M_{4}^{2}}-\frac{m_{Z}^{2}}{M_{4}^{2}}\right)^{2}\right], \\
& \Gamma_{\varphi_{2}^{+} \rightarrow W^{+} Z}= \frac{s_{W}^{4} g^{4}}{16 c_{W}^{2} \pi} \frac{v_{4}^{2}}{M_{4}} \lambda^{\frac{1}{2}}\left(M_{4} ; m_{W}, m_{Z}\right) \\
& \times\left[2+\frac{M_{4}^{4}}{m_{W}^{2} m_{Z}^{2}}\left(1-\frac{m_{W}^{2}}{M_{4}^{2}}-\frac{m_{Z}^{2}}{M_{4}^{2}}\right)^{2}\right],
\end{aligned}
$$

$$
\begin{gathered}
\Gamma_{\varphi_{1}^{+} \rightarrow W^{+} \gamma}=\frac{3 g^{2} e^{2}}{64 \pi} \frac{v_{4}^{2}}{M_{4}}\left(1-\frac{m_{W}^{2}}{M_{4}^{2}}\right), \\
\Gamma_{\varphi_{2}^{+} \rightarrow W^{+} \gamma}=\frac{g^{2} e^{2}}{16 \pi} \frac{v_{4}^{2}}{M_{4}}\left(1-\frac{m_{W}^{2}}{M_{4}^{2}}\right),
\end{gathered}
$$

where the factor $\lambda\left(m_{1} ; m_{2}, m_{3}\right)$ is defined as

$$
\lambda\left(m_{1} ; m_{2}, m_{3}\right) \equiv 1+\frac{m_{2}^{4}}{m_{1}^{4}}+\frac{m_{3}^{4}}{m_{1}^{4}}-\frac{2 m_{2}^{2}}{m_{1}^{2}}-\frac{2 m_{3}^{2}}{m_{1}^{2}}-\frac{2 m_{2}^{2} m_{3}^{2}}{m_{1}^{4}} .
$$

In Fig. 2, we show the BRs for $\varphi^{++}$and $\varphi_{1}^{+}$as a function of the Yukawa coupling $y_{L}$, where we assume only one element of $y_{L_{a b}}$ dominates for simplicity, and we fixed some parameters such as $v_{4}=1 \mathrm{GeV}, M_{\Sigma}=600 \mathrm{GeV}$, and $M_{4}=1000 \mathrm{GeV}$. We find that the BR for the two massive gauge boson mode is dominant if the Yukawa coupling is $y_{L} \lesssim 0.1$, where $W^{+} \gamma$ mode in decay of $\varphi_{1}^{+}$is negligible since it is found to be always less than $\sim 10^{-5}$. The BRs for $\varphi_{2}^{+}$and $\varphi^{0}$ have similar behavior, where $\varphi_{R}^{0}$ can decay into $Z Z$ while $\varphi_{I}^{0}$ cannot decay into a two gauge boson.

The exotic fermions decay into SM lepton and scalar bosons through Yukawa interaction in Eqs. (7) and (13). For $M_{4}>M_{\Sigma}$, the dominant decay modes include only the scalar boson from doublet $H_{1}$ such as $\Sigma^{+} \rightarrow H^{+} \nu$, etc. Here we choose $M_{\Sigma}$ larger than scalar boson masses from doublet fields. On the other hand, $\Sigma$ 's can decay into both scalar bosons from $H_{4}$ and from doublets for $M_{4}<M_{\Sigma}$, where the BRs are determined by relative values of $y_{L}$ and $y_{R}$ Yukawa coupling constants. In general, decay widths of $\Sigma$ 's are given by 
TABLE II. The possible final states from $\Sigma^{+} \Sigma^{-}$and $\Sigma^{\prime+} \Sigma^{\prime-}$ with their fractions given by product of BRs under the assumption of $M_{4}<M_{\Sigma}, y_{L} \gg y_{R}, y_{L} \lesssim 0.1$, and $v_{4}=1 \mathrm{GeV}$.

\begin{tabular}{lcccccc}
\hline \hline From $\Sigma^{+} \Sigma^{-}$ & $\ell^{+} \ell^{-} Z Z Z Z$ & $\ell^{+} \ell^{-} Z Z \varphi_{I}^{0}$ & $\ell^{ \pm} \nu Z Z Z W^{\mp}$ & $\ell^{+} \ell^{-} \varphi_{I}^{0} \varphi_{I}^{0}$ & $\ell^{ \pm} \nu Z W^{\mp} \varphi_{I}^{0}$ & $\nu \nu W^{+} W^{-} Z Z$ \\
\hline Fractions & 0.016 & 0.032 & 0.094 & 0.016 & 0.094 & 0.56 \\
From $\Sigma^{\prime+} \Sigma^{\prime-}$ & $\ell^{+} \ell^{-} W^{+} W^{+} W^{-} W^{-}$ & $\ell^{ \pm} \nu Z W^{\mp} W^{\mp} W^{ \pm}$ & $\nu \nu Z Z W^{+} W^{-}$ & \\
Fractions & & 0.25 & 0.25 & 0.25 & \\
\hline \hline
\end{tabular}

$$
\Gamma_{\Sigma \rightarrow \Phi \ell(\nu)} \simeq \frac{C_{\Sigma \Phi \ell(\nu)}^{2}}{16 \pi} M_{\Sigma}\left(1-\frac{M_{4}^{2}}{M_{\Sigma}^{2}}\right)^{2}
$$

where $\Phi=\left\{\varphi^{ \pm \pm}, \varphi_{1,2}^{ \pm}, \varphi_{R, I}^{0}, h, H, A, H^{ \pm}\right\}$and $\Sigma=\left\{\Sigma^{ \pm}\right.$, $\left.\Sigma^{\prime \pm}, \Sigma^{0}\right\}$ with possible charge combination in final states, and $C_{\Sigma \Phi \ell(\nu)}$ denotes the coupling of an interaction $\Sigma-\Phi-$ $\ell(\nu)$ in Eqs. (7) and (13). We note that the charged component $\Sigma^{ \pm}\left(\Sigma^{\prime \pm}\right)$ can decay into $\Sigma^{0} \pi^{ \pm}$, where $\pi^{ \pm}$is induced from an off-shell $W$ boson since the mass difference between charged and neutral components is induced at the one-loop level [27]. The mass difference is obtained as $\Delta M \sim$ $166 \mathrm{MeV}$ for the triplet fermion and partial decay width is estimated to be $\Gamma_{\pi} \sim 3 \times 10^{-15} \mathrm{GeV}$. This width is much smaller than those in Eq. (41) as we obtain $\Gamma_{\Sigma \rightarrow \Phi \ell(\nu)} \sim$ $10^{-7} \mathrm{GeV}$ with $C_{\Sigma \Phi \ell(\nu)}=10^{-4}, M_{\Sigma}=1000 \mathrm{GeV}$, and $M_{4}=500 \mathrm{GeV}$. We can thus neglect the decay mode with pion in our analysis.

\section{Signals at the LHC}

Here we discuss signals of our model at the LHC focusing on charged particles in the quartet scalar and triplet fermion. The charged scalar boson from $\mathrm{H}_{4}$ dominantly decays into two SM gauge bosons since Yukawa coupling constant $y_{L}$ tends to be much smaller than $\sim 0.1$ to obtain active neutrino mass consistent with observations. Thus signal processes will be

$$
\begin{aligned}
& p p \rightarrow \varphi^{++} \varphi^{--} \rightarrow W^{+} W^{+} W^{-} W^{-}, \\
& p p \rightarrow \varphi_{1,2}^{+} \varphi_{1,2}^{-} \rightarrow W^{+} W^{-} Z Z .
\end{aligned}
$$

Then $W^{ \pm}$and $Z$ bosons further decay into either jets or leptons. For such a signal, detailed discussions are found in, e.g., Refs. [16,28-30]. Then we focus on signals from exotic charged fermion production hereafter.

We consider two cases of mass relation in considering the charged fermion $\Sigma^{ \pm}\left(\Sigma^{\prime \pm}\right)$ : case A, $M_{4}>M_{\Sigma}$ and case $\mathrm{B}, M_{4}<M_{\Sigma}$. In case A, $\Sigma^{ \pm}\left(\Sigma^{\prime}\right)$ always decay into the SM lepton and scalar boson associated with the Higgs doublet. On the other hand, in case B, the charged fermions can decay also into the SM lepton and scalar boson associated with the quartet scalar in addition to the mode in case A. The signals in case A are then obtained as decay modes of $\Sigma^{ \pm}\left(\Sigma^{\prime \pm}\right)$, which are $\ell^{ \pm}\{H, h, A\}$ and $\nu H^{ \pm}$; then scalar bosons further decay into SM fermions or gauge bosons, where the corresponding BRs are the same situation as in the type-I 2HDM. Here we focus on the signals of the SM lepton with a component of $\mathrm{H}_{4}$ in case $\mathrm{B}$, since they are the specific signature of our model. The possible final states from produced $\Sigma^{+} \Sigma^{-}$and $\Sigma^{+} \Sigma^{\prime-}$ pairs are summarized in Table II, with their fractions obtained from the product of BRs for each particle. Here we assume $M_{4}<M_{\Sigma}, y_{R} \ll$ $y_{L} \lesssim 0.1$, and $v_{4}=1 \mathrm{GeV}$ and we do not distinguish neutrino and antineutrino for simplicity; we include decay of components of $\mathrm{H}_{4}$ into SM gauge bosons. Note that we remain $\varphi_{I}$ in the table since it cannot decay into SM gauge bosons, but decays into SM fermions via mixing with the $C P$-odd component in the Higgs doublet sector.

For $\Sigma^{+} \Sigma^{-}$production, we obtain the largest number of events from $\nu \nu W^{+} W^{-} Z Z$ final state. When $W^{ \pm}$and $Z$ bosons from one of $\Sigma^{ \pm}$decay into leptons and the other gauge bosons decay into jets the signal event up to the detector level is given by

$$
p p \rightarrow \Sigma^{+} \Sigma^{-} \rightarrow \varphi_{1}^{+} \bar{\nu} \varphi_{1}^{-} \nu \rightarrow W^{+} Z \bar{\nu} W^{-} Z \nu \rightarrow \ell^{ \pm} \ell^{+} \ell^{-} 4 j E_{T},
$$

where $j$ indicates the jet and $E_{T}$ is the missing transverse energy. Thus our signal is multilepton with jets and missing transverse energy. For $M_{\Sigma}=600 \mathrm{GeV}$, the number of events without kinematical cut can be estimated as $\sim 8$, taking integrated luminosity as $300 \mathrm{fb}^{-1}$. Although the number of events is not large, we would find the signal since the number of the SM background (BG) events is expected to be small. In addition, we can partially reconstruct the mass of $\Sigma^{ \pm}$from $\ell^{ \pm} \ell^{+} \ell^{-} E_{T}^{\prime}$. In the highluminosity LHC (HL-LHC) experiments, we can obtain more events and more parameter regions will be explored.

For $\Sigma^{\prime+} \Sigma^{\prime-}$ production, the most clear signal would come from the final state $\ell^{+} \ell^{-} W^{+} W^{+} W^{-} W^{-}$. When $W^{ \pm}$bosons from one $\Sigma^{\prime \pm}$ decay into leptons and the other gauge bosons decay into jets the signal event up to the detector level is given by

$$
\begin{aligned}
p p & \rightarrow \Sigma^{\prime+} \Sigma^{\prime-} \rightarrow \varphi^{++} \ell^{-} \varphi^{--} \ell^{+} \rightarrow \ell^{-} W^{+} W^{+} \ell^{+} W^{-} W^{-} \\
& \rightarrow \ell^{+} \ell^{-} \ell^{ \pm} \ell^{ \pm} 4 j E_{T}^{\prime} .
\end{aligned}
$$

For $M_{\Sigma}=600 \mathrm{GeV}$, we obtain the production cross section $\sigma\left(p p \rightarrow \Sigma^{\prime+} \Sigma^{\prime}\right) \simeq 7.6 \mathrm{fb}$ as shown in Fig. 1 . The products of production cross section and BRs is then 
$\sigma\left(p p \rightarrow \Sigma^{\prime+} \Sigma^{\prime}\right) \mathrm{BR}\left(\Sigma^{\prime \pm} \rightarrow W^{ \pm} W^{ \pm} \ell^{\mp}\right)^{2} \mathrm{BR}\left(W^{ \pm} \rightarrow \ell^{ \pm} \nu\right)^{2}$ $\mathrm{BR}\left(W^{ \pm} \rightarrow j j\right) \simeq 0.04 \mathrm{fb}$, where we applied $\operatorname{BR}\left(\Sigma^{\prime \pm} \rightarrow\right.$ $\left.W^{ \pm} W^{ \pm} \ell^{\mp}\right) \simeq 0.5, \quad \operatorname{BR}\left(W^{ \pm} \rightarrow \ell^{ \pm} \nu\right) \simeq 0.22$, and $\operatorname{BR}\left(W^{ \pm} \rightarrow\right.$ $j j) \simeq 0.67$. Thus the number of events without kinematical cut can be estimated as $\sim 12$, taking integrated luminosity as $300 \mathrm{fb}^{-1}$. This cross section would be around the same order as the BG process; for example, we obtain the cross section for $p p \rightarrow W^{+} W^{-} \ell^{+} \ell^{-} \rightarrow \ell^{+} \ell^{+} \ell^{-} \ell^{-} \nu \nu$ as $0.18 \mathrm{fb}$ in the SM estimated by MADGRAPH5 [31]. This signature is clearer than the previous case, since the number of events is larger and the final state includes three same sign leptons. In this case, we can partially reconstruct the mass of $\Sigma^{\prime \pm}$ from $\ell^{\mp} \ell^{ \pm} \ell^{ \pm} E_{T}$. However, it is not trivial to select three charged leptons to reconstruct the mass from four charged leptons in the final state and we need to perform detailed analysis. In addition, we need to impose appropriate tagging and kinematical cuts to reduce BG events for getting sufficient significance; for example, jet tagging will be useful to reduce $W^{+} W^{-} \ell^{+} \ell^{-} \mathrm{BG}$ and cuts regarding angles among charged leptons can be used to choose charged leptons as decay products of one $\Sigma^{\prime \pm}$. Furthermore, detector level simulation is required to take into account detector efficiency in order to obtain a realistic number of events at the experiments. The detailed simulation study including BG events and kinematical cuts are beyond the scope of this paper, since the final states contain many particles and the analysis will be very complicated.

\section{SUMMARY AND DISCUSSIONS}

We have constructed a model with hidden $U(1)$ gauge symmetry, which can naturally realize a linear seesaw mechanism by introducing $S U(2)_{L}$ triplet Dirac fermion and quartet scalar fields. Then an induced active neutrino mass is suppressed by two factors: small VEV of a quartet scalar and the inverse of $\mathrm{TeV}$ scale Dirac mass for a triplet fermion, where a small quartet VEV is also required by the $\rho$-parameter constraint. Furthermore, small VEV of the quartet is naturally realized by a mechanism similar to the Higgs triplet model.

We have formulated an active neutrino mass matrix with linear seesaw mechanism, which is given by Yukawa coupling constants associated with interactions among the triplet fermion, quartet scalar, and SM leptons. Then the typical size of Yukawa coupling constants have been estimated to realize the small neutrino mass of $\mathcal{O}(0.1) \mathrm{eV}$. We have found that the size of the coupling can be $\mathcal{O}\left(10^{-5}\right)-\mathcal{O}\left(10^{-4}\right)$ which is similar to those in generating SM charged leptons.

We have discussed collider phenomenology of the model, focusing on production of exotic charged particles. Specific signatures of our model are obtained, when an exotic charged fermion decays into a SM lepton and a scalar boson from the quartet, which dominantly decay into SM gauge bosons. Then our signals are multileptons with jets and missing transverse energy. We have found that the number of signal events is $\mathcal{O}(10)$ at the $\mathrm{LHC} 13 \mathrm{TeV}$ with integrated luminosity of $300 \mathrm{fb}^{-1}$, when triplet fermion mass is $\sim 600 \mathrm{GeV}$. Although the number of events is not large, we may observe the signal since the number of SM background events should be also small for the multilepton final state. In the HL-LHC experiments, we can obtain a larger number of events and a larger parameter region can be explored. More detailed simulation study is left as a future work.

\section{ACKNOWLEDGMENTS}

H. O. is sincerely grateful for the Korea Institute for Advanced Study (KIAS) members. This research is supported by the Ministry of Science, ICT \& Future Planning of Korea, the Pohang City Government, and the Gyeongsangbuk-do Provincial Government (H. O.)
[1] P. Minkowski, Phys. Lett. 67B, 421 (1977).

[2] T. Yanagida, in Proceedings of the Workshop on the Unified Theory and the Baryon Number in the Universe, edited by O. Sawada and A. Sugamoto (KEK, Tsukuba, Japan, 1979), p. 95.

[3] M. Gell-Mann, P. Ramond, and R. Slansky, Supergravity, edited by $\mathrm{P}$. van Nieuwenhuizen et al. (North Holland, Amsterdam, 1979) p. 315; S. L. Glashow, The future of elementary particle physics, in Proceedings of the 1979 Cargèse Summer Institute on Quarks and Leptons, edited by M. Levy et al. (Plenum Press, New York, 1980), p. 687.

[4] R. N. Mohapatra and G. Senjanovic, Phys. Rev. Lett. 44, 912 (1980).
[5] R. N. Mohapatra and J. W. F. Valle, Phys. Rev. D 34, 1642 (1986).

[6] D. Wyler and L. Wolfenstein, Nucl. Phys. B218, 205 (1983).

[7] E. K. Akhmedov, M. Lindner, E. Schnapka, and J. W. F. Valle, Phys. Lett. B 368, 270 (1996).

[8] E. K. Akhmedov, M. Lindner, E. Schnapka, and J. W. F. Valle, Phys. Rev. D 53, 2752 (1996).

[9] A. Das, T. Nomura, H. Okada, and S. Roy, Phys. Rev. D 96, 075001 (2017).

[10] W. Wang and Z. L. Han, Phys. Rev. D 92, 095001 (2015).

[11] K. Kumericki, I. Picek, and B. Radovcic, Phys. Rev. D 86, 013006 (2012). 
[12] S. S. C. Law and K. L. McDonald, Phys. Rev. D 87, 113003 (2013).

[13] Y. Yu, C. X. Yue, and S. Yang, Phys. Rev. D 91, 093003 (2015).

[14] T. Nomura, H. Okada, and Y. Orikasa, Phys. Rev. D 94, 055012 (2016).

[15] W. Wang and Z. L. Han, J. High Energy Phys. 04 (2017) 166.

[16] T. Nomura and H. Okada, Phys. Rev. D 96, 095017 (2017).

[17] T. Nomura and H. Okada, Phys. Lett. B 783, 381 (2018).

[18] W. Konetschny and W. Kummer, Phys. Lett. B 70, 433 (1977); T. P. Cheng and L. F. Li, Phys. Rev. D 22, 2860 (1980); M. Magg and C. Wetterich, Phys. Lett. B 94, 61 (1980); J. Schechter and J. W. F. Valle, Phys. Rev. D 22, 2227 (1980); R. N. Mohapatra and G. Senjanovic, Phys. Rev. D 23, 165 (1981).

[19] C. Patrignani et al. (Particle Data Group), Chin. Phys. C 40, 100001 (2016).

[20] J. A. Casas and A. Ibarra, Nucl. Phys. B618, 171 (2001).

[21] E. Fernandez-Martinez, J. Hernandez-Garcia, and J. LopezPavon, J. High Energy Phys. 08 (2016) 033.
[22] N. R. Agostinho, G. C. Branco, P. M. F. Pereira, M. N. Rebelo, and J. I. Silva-Marcos, Eur. Phys. J. C 78, 895 (2018).

[23] D. V. Forero, M. Tortola, and J. W. F. Valle, Phys. Rev. D 90, 093006 (2014).

[24] A. M. Baldini et al. (MEG Collaboration), Eur. Phys. J. C 76, 434 (2016).

[25] A. Belyaev, N. D. Christensen, and A. Pukhov, Comput. Phys. Commun. 184, 1729 (2013).

[26] P. M. Nadolsky, H. L. Lai, Q. H. Cao, J. Huston, J. Pumplin, D. Stump, W. K. Tung, and C.-P. Yuan, Phys. Rev. D 78, 013004 (2008).

[27] M. Cirelli, N. Fornengo, and A. Strumia, Nucl. Phys. B753, 178 (2006).

[28] F. del Aguila, M. Chala, A. Santamaria, and J. Wudka, Phys. Lett. B 725, 310 (2013).

[29] F. del Águila and M. Chala, J. High Energy Phys. 03 (2014) 027.

[30] M. Chala, C. Krause, and G. Nardini, J. High Energy Phys. 07 (2018) 062.

[31] J. Alwall, R. Frederix, S. Frixione, V. Hirschi, F. Maltoni, O. Mattelaer, H.-S. Shao, T. Stelzer, P. Torrielli, and M. Zaro, J. High Energy Phys. 07 (2014) 079. 